\title{
Modifying the IEEE 802.11 MAC to improve performance of multiple broadcasting of multimedia data in wireless ad-hoc networks
}

\author{
Christos Chousidis \\ School of Engineering and Design \\ Brunel University \\ London, UK
}

\author{
Rajagopal Nilavalan \\ School of Engineering and Design \\ Brunel University \\ London, UK
}

\begin{abstract}
Multimedia applications over wireless networks have dramatically increased over the past years. Numerous new devices and applications that distribute audio and video over wireless networks are introduced every day and all of them demands a reliable and efficient wireless standard. Either operating as independent ad-hoc networks or as terminal parts of wired networks or the internet, wireless networks are frequently facing the need to broadcast multimedia data from multiple sources to multiple users. IEEE 802.11 standard (Wi-Fi) is the primary technology in wireless networking today. However, it has some inherited problems when it comes to broadcasting caused mainly by the lack of an acknowledgment mechanism. These problems do not allow the standard to take full advantage of the bandwidth offered by its latest amendments. In this paper two independent modifications of the medium access control (MAC) mechanism of the standard, are proposed along with the expanded use of the CTS-to-Self protection mechanism. The main objective of this study is to explore the ability of the modified MAC mechanisms to improve broadcasting performance while are operating in conjunction with a regular wireless network, and also to define the cases were the use of CTS-to-Self-protection mechanism can improve the overall performance of the network. The results show that the overall performance can be improved using these alternative MAC methods. Also, the cases where the CTS-to-Self technique can additionally contribute to the network performance are defined and analyzed.
\end{abstract}

Keywords - wireless networks; broadcasting; multimedia; linear increase of $C W$; EBNA; CTS-to-Self

\section{INTRODUCTION}

The IEEE 802.11 standard (Wi-Fi) is the main technology in wireless networking and its use has an exponential raise [1]. Every day more and more devices and application are adopting this standard and many of them are using this networking platform to transmit multimedia type data [2][3]. Internet TV and radio, VoIP, video conferencing and telepresence, network gaming, and live audio networking are some of the recreational and professional applications that demand reliable and efficient wireless networks [4][5]. Many of those applications are using ad-hoc networking practices and broadcasting or multicasting transmission in order to stream their data. Broadcasting is a good practice in media networking because it can distribute simultaneously data to multiple users. IEEE 802.11 standard supports broadcasting without any type of feedback (e.g. acknowledgment, ACK) from the recipients. Therefore, broadcasting does not provide any kind of delivery guarantee which in some cases, like live media transmission, is not critical as the retransmission of real-time data creates additional problems when it comes to delay and jitter. IEEE 802.11 implements a carrier sense multiple access mechanism, with collision avoidance (CSMA/CA). However, collisions are still happening and due to the lack of ACK, are leading to lost data. CSMA/CA mechanism uses a random backoff technique to reduce the probability of collisions and fairly arbitrate the wireless medium by allocating random waiting time values from a predefined contention window $(\mathrm{CW})$ to every station intended to transmit. The lack of positive ACK of a transmitted packet causes an exponential increase of the $\mathrm{CW}$ and therefore a decrease in the probability of collision. In broadcasting however, as long as ACK is not implemented, the $\mathrm{CW}$ remains constant and always holds the minimum value. In the case of saturated networks and multiple broadcasting environments, the probability of collision is increased; affecting negatively the network's throughput [2]. An additional technique used by the CSMA/CA mechanism to arbitrate the medium access is the distribution of the network allocation vector (NAV) which contains information about the time that the network will be occupied by the station (STA), who recently gained access to the wireless medium. This technique demands an exchange of Request-to-Send, Clear-to-Send control messages (RTS/CTS) which again requires a unique recipient and therefore cannot be implemented in broadcasting. CTS-to-Self control message is an alternative to RTS/CTS process used in the cases were a CTS is not possible. It is broadcasted from a station with destination address its own address and lower transmission rate. This technique is strictly used as protection mechanism only for mixed-mode environments where extended rate physical (ERP-802.11g) and/or high throughput (HT-802.11n) devices coexist with legacy 802.11 technologies [6]. As long as ACK and NAV distribution is not implemented in broadcasting, saturated media broadcasting STAs are suffering from two main problems: large number of collisions and data loss due to buffer overflow caused by the excessively busy wireless medium.

To address the above problems two modifications of the IEEE 802.11 MAC mechanism are proposed in this paper. The amendments focus on two main areas, the random backoff algorithm and the NAV distribution. The IEEE 802.11 MAC is 
reprogrammed to send a CTS-to-Self message prior to every broadcasting packet using the operational-high data rate. The random backoff process is also modified using two different approaches. In the first one, a linear increase of the $\mathrm{CW}$ is applied taking into account the changes in the number of broadcasting STAs. In the second approach, again a linear increase of the $\mathrm{CW}$ is performed according to the variation of the broadcasting STAs, but in this case an exclusive backoff number allocation (EBNA) algorithm is implemented. This algorithm allocate exclusive backoff numbers to each STA while maintain fairness in waiting periods between STAs over the time.

The main objective of this study is to explore the ability of the modified MAC mechanisms to improve broadcasting performance while are operating in conjunction with a regular wireless network. For this reason, the above modifications are tested in a "mixed" network where broadcasting and unicasting STAs coexist. The performance of the network is studied as a whole in order to investigate the possibility of different MAC mechanisms to function together.

The remaining of this paper is organized as follows: In section II, the 802.11 MAC process is summarized and the drawbacks of random backoff algorithm in the case of multiple broadcasting are analysed. In section III, the proposed modifications of the MAC process and the use of CTS-to-Self protection mechanism, are thoroughly described. In Section IV, the simulation's characteristics are analysed and comparative results are presented and commented while in Section V, the conclusions of this work are presented.

\section{ANALYSIS AND DRAWBACKS OF IEEE 802.11 MEDIUM ACCESS MECHANISM}

\section{A. General description}

The IEEE 802.11 MAC is mainly designed for wireless unicast communication and for unlimited number of users in the network. In Distributed Coordination Function (DCF) which is its primary medium arbitration method, Random Backoff in conjunction with virtual and physical carrier sense provides a level of protection from collisions. The 802.112007 standard provide an additional protection mechanism using RTS/CTS or CTS-to-Self control frames. This is mainly used for Network Allocation Vector (NAV) distribution in mixedmode environments where different 802.11 technologies coexist. Although RTS/CTS it is used to address the hidden node problem, CTS-to-Self is used strictly as a protection mechanism for mixt-mode networks using data rates and modulation method that legacy 802.11 technologies can understand. NAV is distributed by setting the duration field of the control frame with the time in microseconds required in order for the two parties to complete transmission including ACK. It is clear however that there is no MAC-Level recovery mechanism in broadcasting [7]. In multimedia broadcasting the focus must be on preventing the loss of packets and the collisions instead of recovery and retransmission. NAV distribution is possible in broadcasting, only in mixed mode networks, by using the CTS-to-Self control frame [4]. CTS-toSelf is a standard CTS frame transmitted with a destination address of the transmitting station. The transmitting STA cannot hear its own transmission in a half-duplex medium but all nearby STAs are alerted that a frame broadcast is pending and they can also update their NAVs with the value included in the duration field of the CTS-to-Self frame. As mentioned above, the use of CTS-to-Self is strictly limited in mixed-mode environments and it is using lower data rates that reduce throughput and increase delay. The possibility of modifying the 802.11 MAC to use CTS-to-Self as a main NAV distribution method, using also high data rates will significantly contribute to the performance of the protocol especially in broadcasting. However, the use of CTS-to-Self alone cannot eliminate the collisions occurrence which is caused by the drawbacks of 802.11 MAC Random Backoff mechanisms. This mechanism significantly contributes in collision avoidance but cannot totally eliminate them, especially when the number of STAs increases. In heavy data loads, there is a high likelihood that two or more STAs will choose the same backoff value. In this case the collision cannot be avoided regardless of the use of CTS-to-Self. For this reason an alternative EBNA algorithm can be used to overcome the Random Backoff algorithm drawbacks in the case where multiple broadcasting is taking place

\section{B. Analysis of IEEE 802.11 MAC algorithm}

IEEE 802.11 MAC Layer is the lowest part of the Link Layer and it is placed between the Physical (PHY) and the Logical Link Control (LLC) sub-layer. MAC architecture is based on two basic coordination functions, Point Coordination Function (PCF) and Distributed Coordination Function (PCF). PCF is a contention free access method which provides polling intervals to allow uncontended transmission opportunities (TXOP) for participating STAs. This function is outside the scope of this paper, first because it demands the use of an AP and second, because the manufacturers never implemented it into their devices. In this study the fundamental DCF contention-based access mechanism is used.

DCF's timing diagram is illustrated in figure 1 and its function is described as follow. A STA with a packet to transmit waits for the channel to become idle. When an idle period equal to DCF Inter-Frame Space (DIFS) is detected, generates an initial Backoff time value. This value indicates the period that the STA has to additionally defer before transmitting. The random Backoff process is the most important mechanism used in IEEE 802.11 CSMA/CA to prevent collisions. CW increases exponentially for every retransmission, (unique per station). Under low utilization, stations are not forced to wait very long before transmitting their frame. If the utilization of the network is high, the protocol holds stations back for longer period of times to avoid the probability of multiple stations transmitting at the same time. When we are referring to Contention-Based access, random Backoff is actually the primary mechanism for contention. This value is extracted from the following formula:

$$
\text { Backoff_Time }=\operatorname{INT}(C W \times \text { Random }(0,1)) \times \text { aSlotTime }
$$

Random $(0,1)$ is a pseudo-random number between 0 and 1 drown from a uniform distribution. $\mathrm{CW}$ is an integer within the range of values $\mathrm{CWmin}$ and $\mathrm{CWmax}$. CWvalues $=2 \mathrm{x}-1$ (x starts from an integer defined by the station and goes up to 10). For example, for $\mathrm{x}=4, \mathrm{CW} 4=24-1=15, \mathrm{CW} 5=31, \mathrm{CW} 6=63 \ldots \ldots$ $\mathrm{CW} 10=1023$. The aSlotTime duration is the value of the 
correspondingly named PHY characteristics. The Backoff timer is decremented with one slot as long as the channel is idle. When a transmission is detected, the Backoff timer freezes and start to decrease again when the channel is sensed idle for a DIFS. When the timer reaches zero the data packet is finally transmitted.

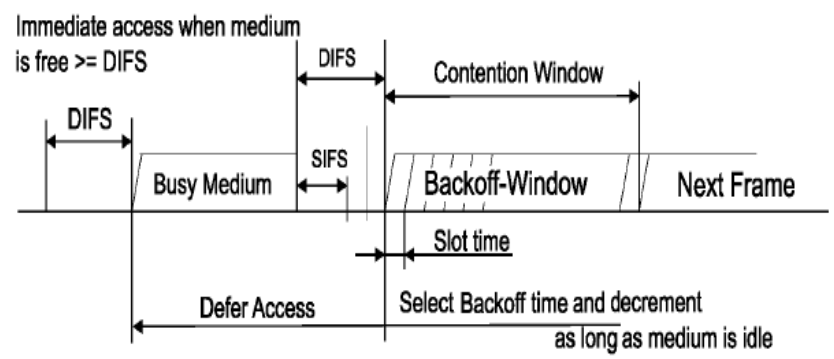

Fig. 1. IEEE 802.11 basic access method

\section{Drawbacks of random backoff in 802.11 broadcasting}

There is plenty of research on the Reliable Broadcasting over wireless ad-hoc networks and many protocols have been proposed [8] [9] [10]. These protocols can be divided into four main categories according to the methods they use.

1) Simple Flooding Methods: Requires each node to retransmit all packets

2) Probability Based Methods: Use some basic understanding of the network topology in order to assign a probability to a node to rebroadcast.

3) Area based Methods: Rebroadcasting is based on the possible additional area that will be covered.

4) Neighbour Knowledge Methods: Maintain a state of neighbours, obtained by "Hallo" messages. This stage is used in the decision to retransmit

All the above methods require a sort of retransmission which is unsuitable for media networking. Reliability in media broadcasting is reduced by the drawbacks of random backoff process, which cause channel access delay and collisions no matter the available bandwidth of the wireless technology that is used.

The IEEE 802.11 standard defines that the CW size exponentially increases for each retransmission attempt of the same packet. However, as there is no retransmission in broadcasting, the CW size always holds the CWmin value. Under high utilization due to the increasing number of STA and/or high data production, CWmin appears to be extremely small. In this case we are facing two major problems. The first one is that it is possible for a STA that just completed a transmission and has a new packet to send, to choose zero as its initial backoff time and start transmitting immediately after a DIFS. As we can see from (1), backoff time is a random outcome based on a uniform distribution but its range increases proportionally with the size of $\mathrm{CW}$. This consecutive transmission will give other STAs no chance to backoff. This problem is refereed as the backoff counter consecutive freeze process (CFP), and was extensively analysed by Xianmin Ma and Xianbo Chen in [11]. They show that the solution would be the ability to increase $\mathrm{CW}$ in broadcasting. The second and most significant problem in the case of wireless media broadcasting is that there is a high likelihood for two or more STAs to choose concurrently equal backoff value. It is easy to understand that when we have fifty or more STAs producing continuous data and they are performing the backoff process using a $\mathrm{CW}=15$ (like in 802.11g \& 802.11n) this is highly possible. In this case a collision is occurring and a data packet is lost as there is no recovery mechanism and no time for retransmission.

For a given size of $\mathrm{CW}$ and " $\mathrm{N}$ " number of STAs broadcasting in a wireless network, the probability " $p$ " of collision is given by the equation:

$$
p=1-\left(1-\frac{1}{C W}\right)^{N-1}
$$

Figure 2 shows the calculated and simulated probability of collision in a multiple broadcasting environment using various CW sizes. A series of simulations performed for this study using OPNET modeler. The IEEE 802.11 MAC was modified to allow broadcasting using different than the minimum, CW sizes. CW size takes the values: 16, 32 and 64. The simulation parameters are: Packet Size 1024 bytes, Physical Characteristics $802.11 \mathrm{~g}-24 \mathrm{Mbps}$, Bit Rate $400 \mathrm{Mbps}$. As we can see from figure 2, for a $\mathrm{CW}=16$ which is the broadcasting CW size according to the standard, the probability of collision for saturated network of 15 broadcasting STAs reach the value of $50 \%$ and approaches $100 \%$ when 50 STAs are broadcasting simultaneously.

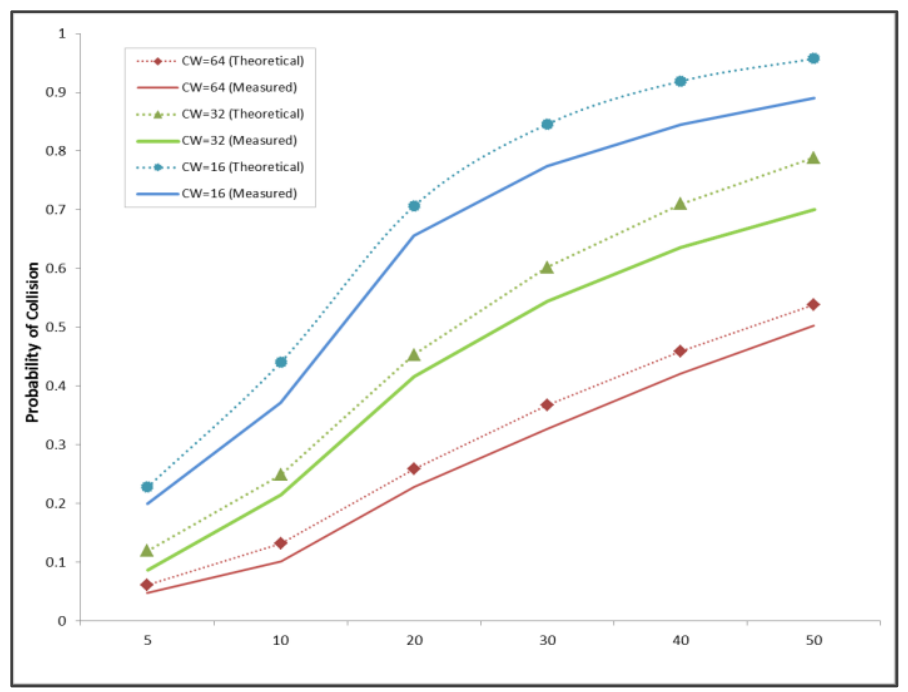

Fig. 2. Probability of Collision

\section{MODIFIED IEEE 802.11 MAC MECHANISM}

As it mentioned earlier in this paper in order to override the inability of the 802.11 protocol in handling multiple broadcasting media data, a modified MAC mechanism is proposed. The amendments focus on two main areas, the NAV distribution and the random Backoff algorithm.

\section{A. Linear increase of $C W$}

According to the IEEE 802.11 standard, in broadcasting the $\mathrm{CW}$ remain unchanged and always hold its minimum 
value. This results in busy networks for broadcasting STAs to significantly increases the probability of collision (Fig 2). In this modification, the $\mathrm{CW}$ dynamically change according to variation of the broadcasting STAs in the network. For this purpose a variable (No_of_STAs) indicating the number of broadcasting STAs is created. The number of Backoff slots is a random value selected using a uniform distribution from a $\mathrm{CW}$ which is given by the formula using:

$$
C W=C W m i n+N o \_o f \_S T A s
$$

\section{B. Exclusive Backoff Number Allocation algorithm (EBNA)}

In order to prevent STAs from choosing similar Backoff numbers which leads to a collision an EBNA algorithm is implemented [12] [13]. This algorithm increases the size of CW according to the number of broadcasting STAs in the network. It is also designed to maintain fairness while allocating exclusive Backoff values for each transmission attempt. In order to do this, the algorithm needs two external variables, the total number of STAs in the wireless network (No_of_STAs) and the Station ID (STID) that every STA obtains upon joining the network (fig:3, line $1 \& 2$ ). The $\mathrm{CW}$ is always given by:

$$
C W=N o \_o f \_S T A s * 2
$$

The algorithm divides the $\mathrm{CW}$ in two equal groups. Values in the groups are allocated as follow:

$$
\begin{aligned}
& \text { group } 1 \leqslant N o \_o f \_S T A s / 2 \\
& \text { group } 2>N o \_o f \_S T A s / 2
\end{aligned}
$$

For each transmission attempt a random value between 1 and 2 is generated in order to select one of the two groups (fig:3, line 3). If group1 is selected the algorithm allocates to the STA a Backoff value equal to its STID, in other case the value given by the algorithm it is a projection of the STID value to group 2 and it is given by the formula:

$$
\text { Backoff_slots }=[(\text { No_of_STAs } * 2)-S T I D]+1
$$

For a network with 10 STAs the station with STID=2 the Backoff_slot variable will take randomly one of the backoff values 2 or 19 while a station with $\mathrm{STID}=6$ will take the Backoff value 6 or 15 (fig. 4). The pseudo-code describing the above process is illustrated in figure 3 .

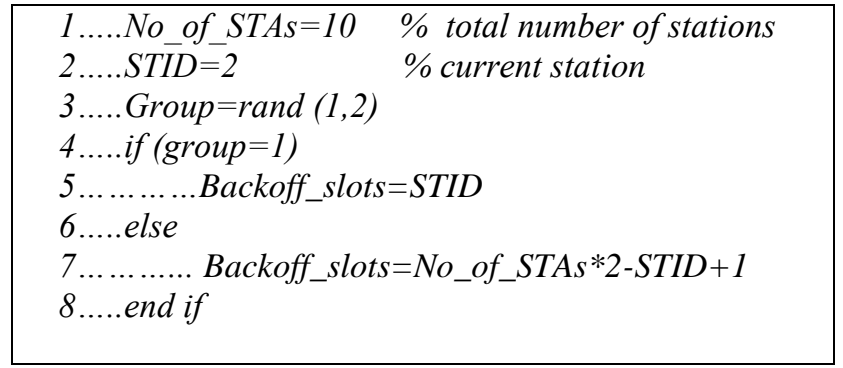

Fig. 3. Exclusive Backoff Number Allocation algorithm pseudo-code

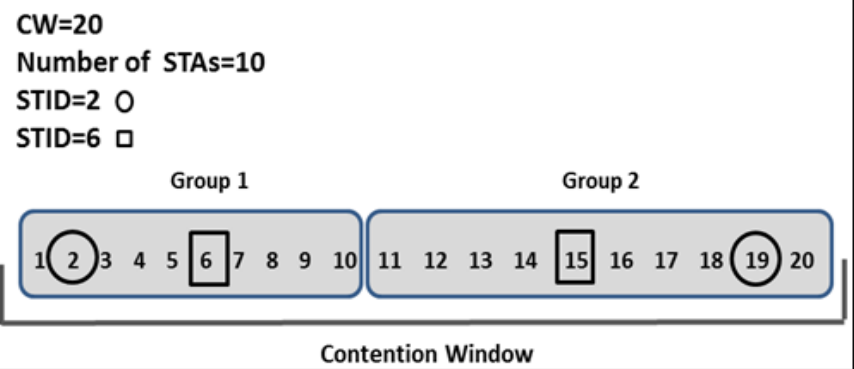

Fig. 4. EBNA example

Figure 5 shows a 3D snapshot of the Backoff number allocation process for both modifications, while figure 6 shows the fairness of the EBNA algorithm over the time as it is illustrated for to different broadcasting STAs during the same simulation by presenting the mean average of Backoff values for these STAs. As we can see, when the network reaches the steady state (approximately $5 \mathrm{sec}$ ) the average waiting time is equal for both STAs.

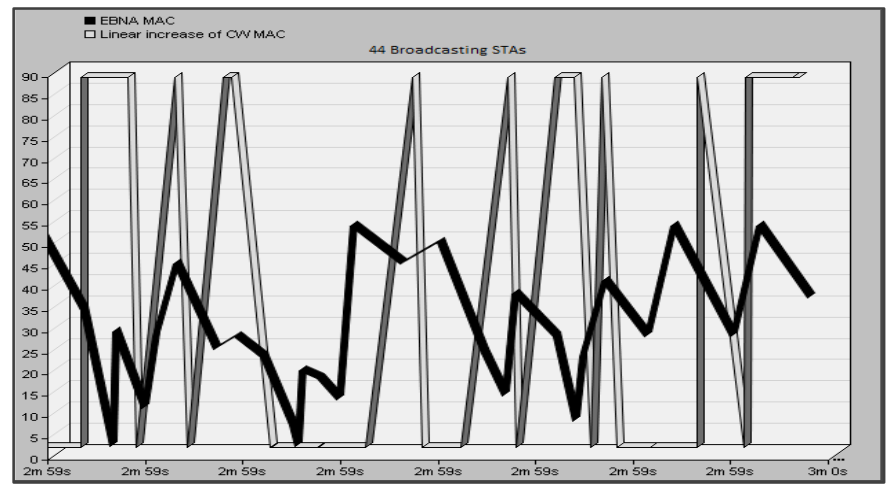

Fig. 5. backoff number allocation process

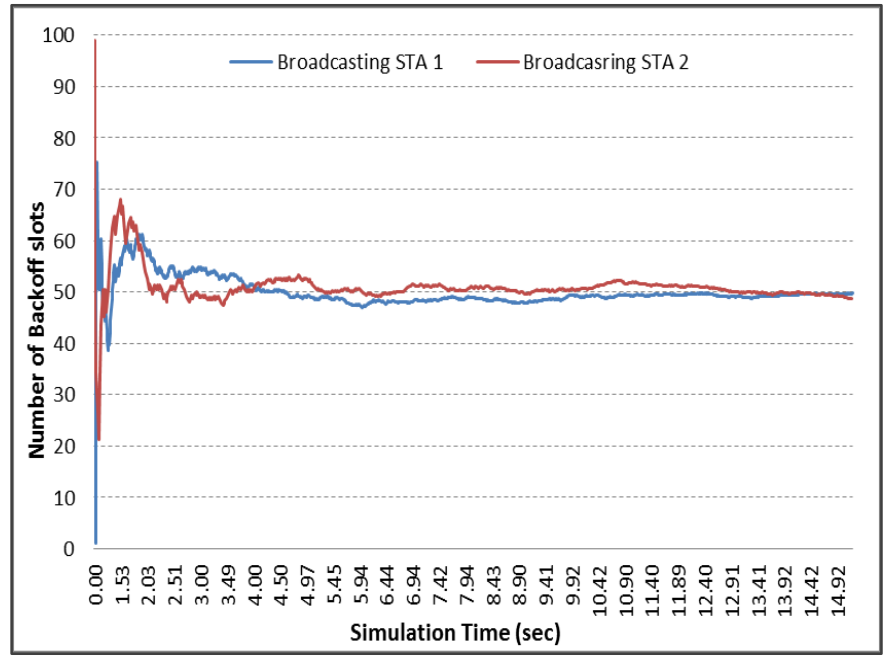

Fig. 6. Fairness in the long run, using the EBNA algorithm 


\section{CTS-to-Self modofication}

CTS-to-Self control message is an alternative to RTS/CTS process currently used in broadcasting only in cases where legacy technologies coexist with an ERP (802.11g) or HT (802.11n) physical, (mixed-mode networks). It is achieved by sending a CTS-to-Self control frame in appropriate (usually lower) data rate and modulation that all STAs can understand. CTS-to-Self frame contains in its "duration" field the time that all non-transmitting STAs must defer before trying to access the medium.

In order for the CTS-to-Self to be used as the main protection mechanism in broadcasting, two major modifications have been done in the 802.11 MAC algorithm. First, while the structure of the CTS-to-Self packet format remained as it was described in the standard; the MAC has been reprogrammed in order to transmit a CTS-to-Self control message prior to every data transmission. Thus, when a STA complete its random Backoff countdown and finds the medium idle, instead of broadcasting a data packet, sends a CTS-to-Self control packet which contains information concerning the time period that the medium will be reserved due to the forthcoming data packet transmission. In the unfortunate event that another STA completes the random Backoff simultaneously, the two CTS-to-Self packets are colliding causing a jam in the network lasting significantly less than a data packet collision (Fig 7).

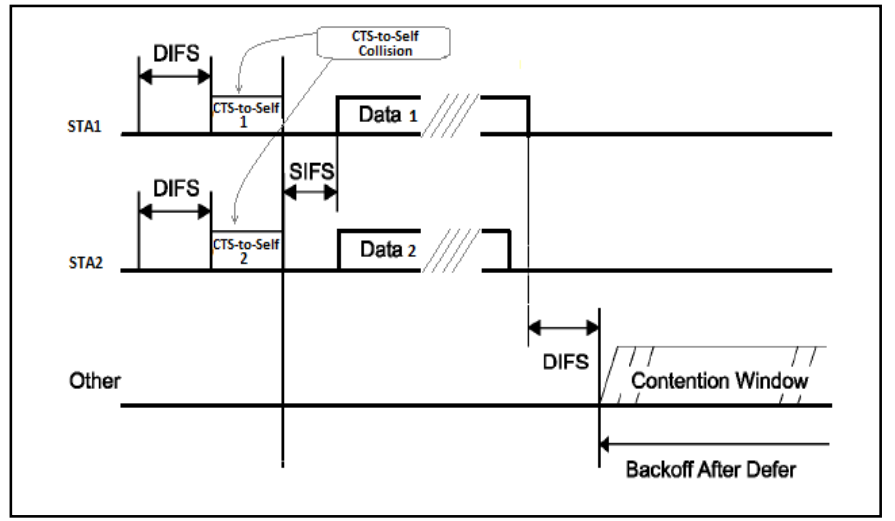

Fig. 7. CTS-to-Self and data Collisions

In any other case the STA waits for a sort inter frame period (SIFS) and transmits the data packet. Another modification in the MAC algorithm, which has been done in this project, was to reprogram the transmission rate of the CTSto-Self message. CTS-to-Self transmission parameter has been modified to always adjust with the selected data rate used for data transmission.

\section{SimUlATION CHARACTERISTICS AND RESUlTS}

\section{A. Simulation characteristics}

The network simulation platform used in this study is OPNET Modeler 17.1. The simulation is based on IEEE $802.11 \mathrm{~g}$ PHY, with a bit rate of 54Mbps. The topology is based on an ad-hoc network in a single BSS, with 56 unicast STAs located in the middle and broadcasting STAs randomly surrounding the unicast group in a $50 \times 50 \mathrm{~m}$ surface. The number of broadcasting STAs is gradually increased from 4 to 44 , taking sequentially the values $4,8,16,24,34$, and 44 . The simulation duration is $3 \mathrm{~min}$. This is enough time for the system to reach its steady state. Three separate simulations have been conducted where all stations were relocated and also a different seed number has been set during the simulation execution. The presented results are the average values, in those cases where significant differences occurred.

All data traffic generation parameters for unicast and broadcast traffic are listed in table 1.

TABLE 1: TRAFFIC GENERATION PARAMETERS

\begin{tabular}{|l|l|}
\hline \multicolumn{2}{|c|}{ Unicast traffic } \\
\hline Start Time & Normal Distribution $(0.5,0.1)$ \\
\hline On-State & $180 \mathrm{sec}$ \\
\hline Off-State & $0 \mathrm{sec}$ \\
\hline $\begin{array}{l}\text { Interarrival } \\
\text { Time }\end{array}$ & Normal Distribution $(0.1,0.005)$ \\
\hline Packet Size & 2200 bytes \\
\hline \multicolumn{2}{|c|}{ Broadcast traffic } \\
\hline Start Time & Normal Distribution $(1,0.01)$ \\
\hline On-State & $180 \mathrm{sec}$ \\
\hline Off-State & 0 sec \\
\hline $\begin{array}{l}\text { Interarrival } \\
\text { Time }\end{array}$ & Constant $(0.0243)$ \\
\hline Packet Size & 1100 bytes \\
\hline
\end{tabular}

Figure 8 shows the network configuration for a population of 56 unicast and 44 broadcast STAs. The resulting load transmitted by each broadcasting STA is approximately $370 \mathrm{Kbps}$ while unicast STAs are transmitting with a bit rate of $77 \mathrm{Kbps}$. The 56 unicast STAs are sending data to randomly chosen destinations including the existing broadcasting STAs. That cause to the broadcasting stations an additional load due to the ACK messages they have to transmit when a unicast packet is addressed to them. All broadcasting STAs are set to unlimited buffer size.

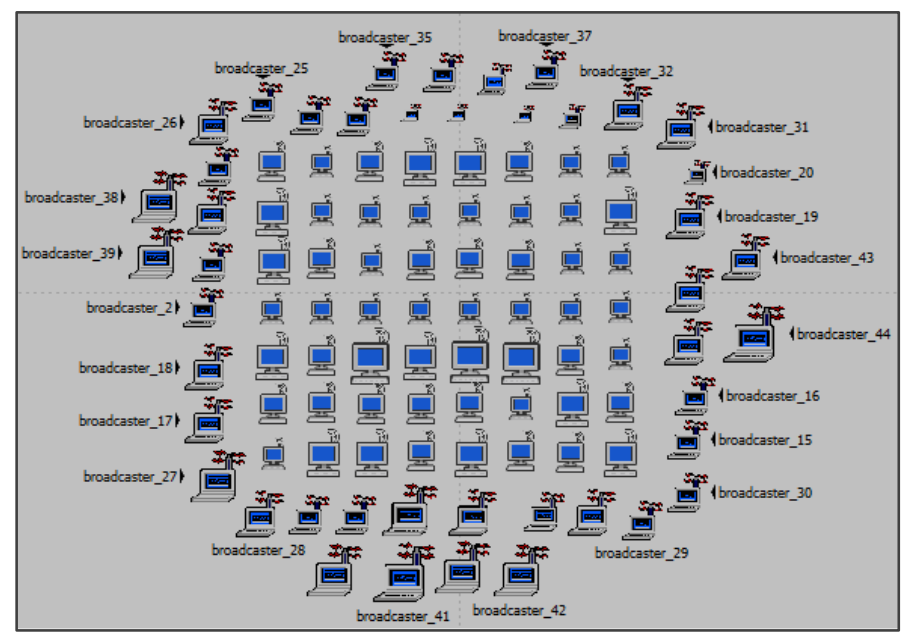

Fig. 8. Network configuration

\section{B. OPNET code modifications}

All modifications to the standard IEEE 802.11 MAC were made using OPNET Modeller network simulation environment. OPNET modeller is a powerful simulation tool which allows users to have full access to the executed code and 
gives the ability to create and modify complex communication protocols. It has its own $\mathrm{C}++$ library and it is using state machines to design and implement processes. For our implementation the OPNET wireless station node model is used.

\section{a) Random Backoff modifications}

Both cases described in III A and B, are implemented in OPNET by modifying the 'wlan dispatch' process and the 'wlan_mac' child process. In the wlan_mac process (BKOFF_NEED state), the Backoff slots allocation algorithm has been changed according to the above described logic. The No_of_STAs variable is entered manually in the source code. For the STID parameter, a custom attribute is created and it is set during the "run" process. It is important to mention here that OPNET use the same "wlan_dispatch" for all models running the IEEE 802.11 standard. In our case, two MAC algorithms has to be used simultaneously; the classic 802.11 MAC used by the unicast STAs and the modified 802.11 MAC used by the broadcasting STAs. For this reason, a modified 'wlan_dispatch' was also created.

\section{b) CTS-to-Self modifications}

A number of modification have been made mainly in Function Block of the wlan_mac process in order to force this modified child process to create a CTS-to-Self message prior to each data packet transmission using also the operational bit rate which in this case is 54 Mbps.

\section{Organising the simulation}

In order to study the performance of the modified MAC algorithms proposed in this paper and also to investigate the effect of the modified CTS-to-Self protection mechanism, five independent simulations have been carried out for each increase of the broadcasting STAs population. The number of unicast STAs as well as their traffic generation parameters remains unchanged during all simulations. Broadcasting STAs are altering their MAC algorithm according to the following combinations:

- Broadcasting using the classic IEEE 802.11 MAC

- Broadcasting using the Linear Increase of CW without CTS-to-Self

- Broadcasting using the Linear Increase of CW with the use of CTS-to-Self

- Broadcasting using the EBNA algorithm without the use of CTS-to-Self

- $\quad$ Broadcasting using the EBNA algorithm with the use of CTS-to-Self

\section{Results}

\section{a) Throughput}

Figure 9 shows the throughput performance for the five different simulations described above. Throughput is measured as the overall load of successfully transmitted data. It is important to note that in the case of broadcasting, a successfully transmitted packet it appears to be measured multiple times, depending on the number of recipients in the wireless network.
Thus, if $n$ is the total number of STAs in the network and $A i$ is the successfully transmitted load from each STA, the overall measured throughput is given by the formula:

$$
\text { Throughput }=(n-1) \sum_{i=0}^{n} A i
$$

As it is shown from figure 9, when the number of broadcasting STAs is small all methods are performing equally. When the number of Broadcasting STAs increases all modified medium access processes are performed slightly better than the classic 802.11 MAC. As it is expected, the EBNA algorithm gives the best results because it guarantees that there are no collisions between broadcasting STAs. All collisions happening at this stage are between broadcasting and unicasting traffic.

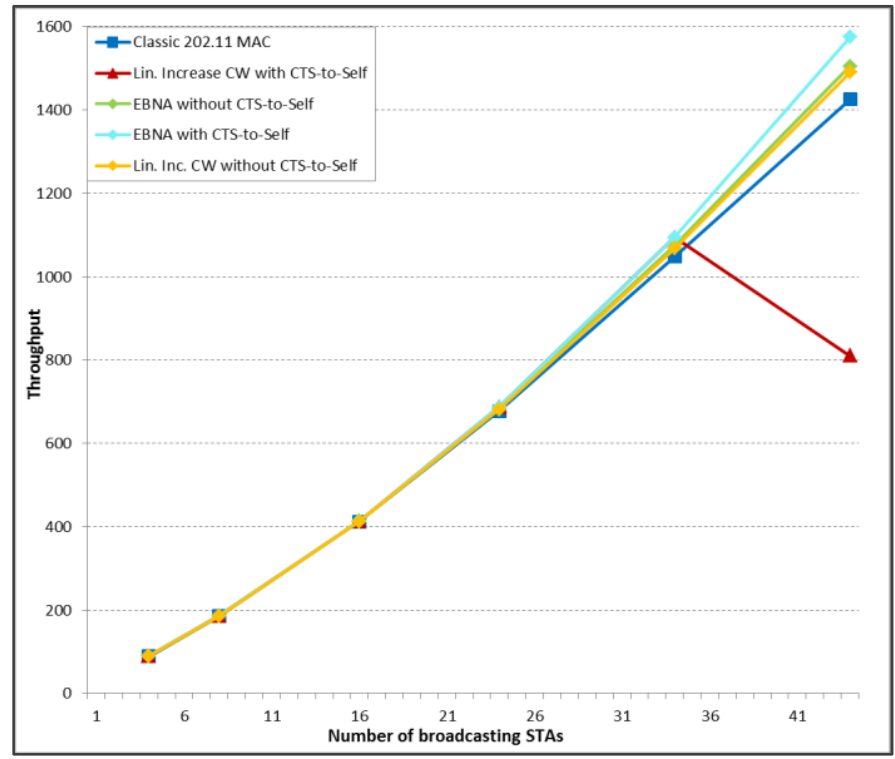

Fig. 9. Overall Throughput

The EBNA with the use of the CTS-to-Self protection mechanism it performs better than the linear increase without CTS-to-Self. This is happening because although the EBNA guarantees no collision between broadcasting traffic, the additional traffic of the CTS-to-Self collides with the unicast packets. Finally, we can see that when linear increase of $\mathrm{CW}$ is used in conjunction with CTS-to-Self, the additional traffic created by this message leads to a network breakdown due to buffer overflow

\section{b) End-to-End delay}

This statistic shows the overall end-to-end delay for the entire network. As it is expected, the delay increases in all modified MAC processes because in all of them a wider $\mathrm{CW}$ it is used. It remains however in acceptable levels. Classic 802.11 has the lowest delay which however represents a smaller number of successfully delivered packets. Figure 10 shows the overall end-to-end delay for all the five cases. Here again, in the linear increase of CW with the use of CTS-to-Self when the number of broadcasting STAs increases the network breaks down and the delay become prohibitively high. 


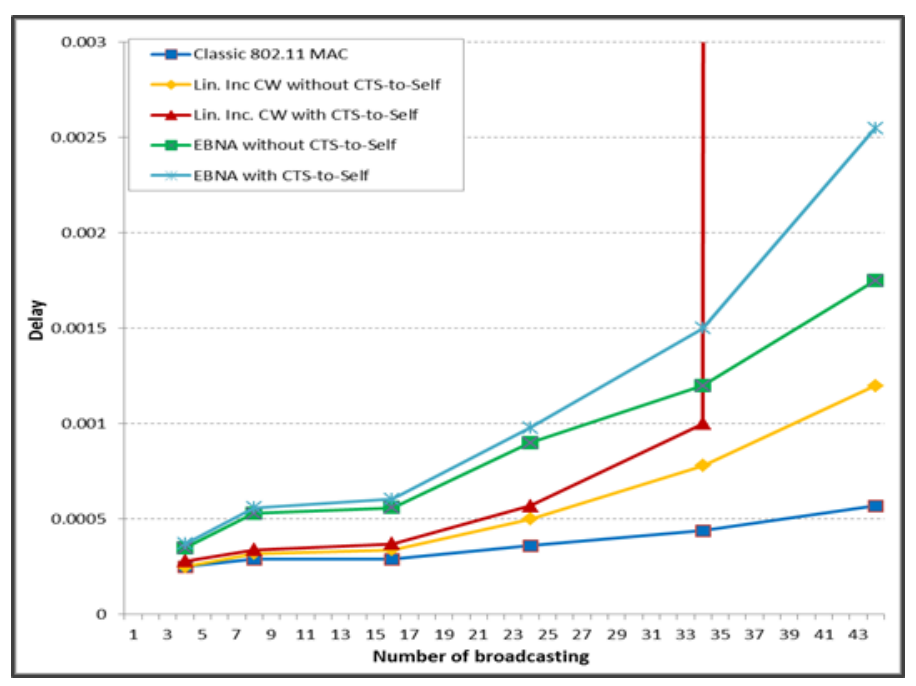

Fig. 10. End-to-End delay

\section{c) Retransmission attempts}

This statistic measures the average retransmission attempts for each packet for the entire network. It is directly affected by the throughput, and as we can understand concerns only the unicast transmission. Broadcasting STAs have no chance for retransmission. Figure 11 shows the retransmission attempts for the overall network

\section{d) Backoff Slots}

The average number of backoff slots is measured in this statistic. As it is mentioned above, there are two different types of MAC algorithms running simultaneously in all simulations. The unicasting STAs use the classic 802.11 MAC and the broadcasting STAs which comply with the cases described in section IV, C. Therefore, two separate statistics for the number of backoff slots are collected in this study. Figure 12 shows the average backoff slots measured in each Broadcasting STA. Figure 13 shows the average backoff slots measured in each Unicasting STA.

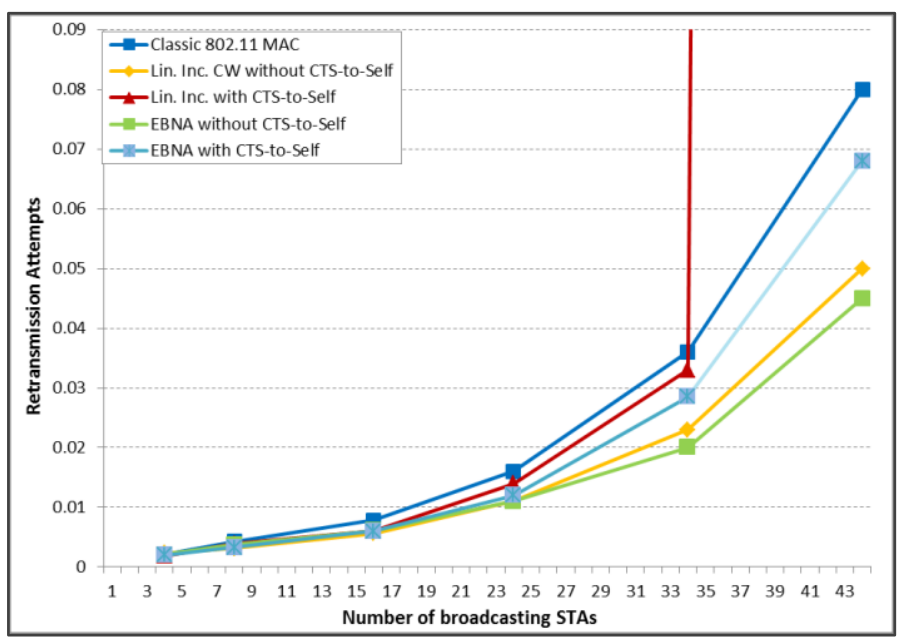

Fig. 11. Retransmission Attempts

In broadcasting, the use of the EBNA algorithm cause longer waiting times mainly due to the bigger size of the $\mathrm{CW}$. The linear increase of $\mathrm{CW}$ gives acceptable low number of backoff slots up to 34 broadcasting STAs. For bigger number of STAs, the network collapses and in the case that CTS-toSelf is used, the number of backoff slots increases dramatically. An interesting observation in the graph of figure 12 is that the increase of the number of backoff slots is not vertical. That gives the evidence that network's breakdown take place in the unicasting and not in broadcasting STAs.

In the unicasting STAs (Fig. 13) where the classic 802.11 MAC is used and also the generated traffic is lower, the number of backoff slots is significantly low and uniform. Exception is the case of linear increase of $\mathrm{CW}$ window with the use of CTS-to-Self where the network collapses.

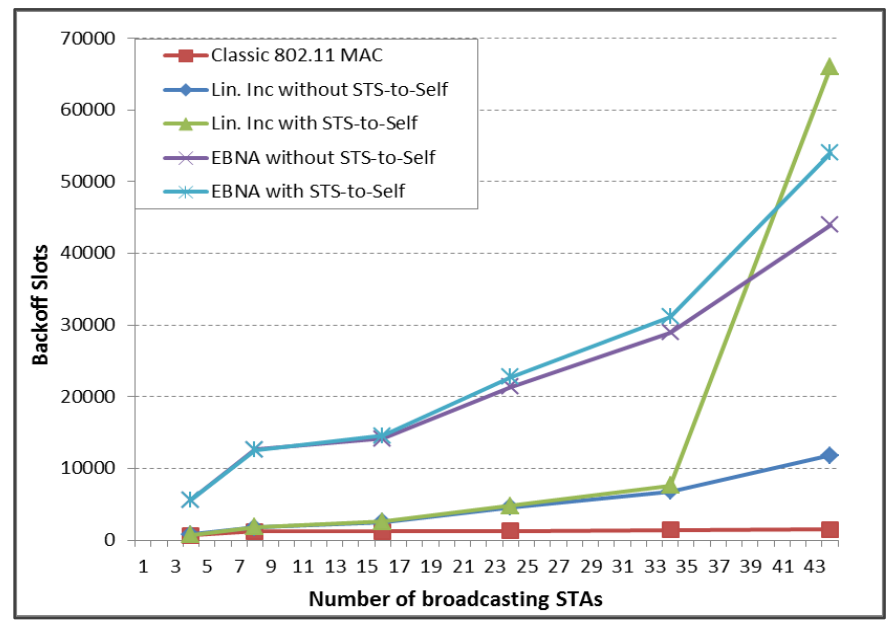

Fig. 12. Number of backoff slots for Broadcasting STAs

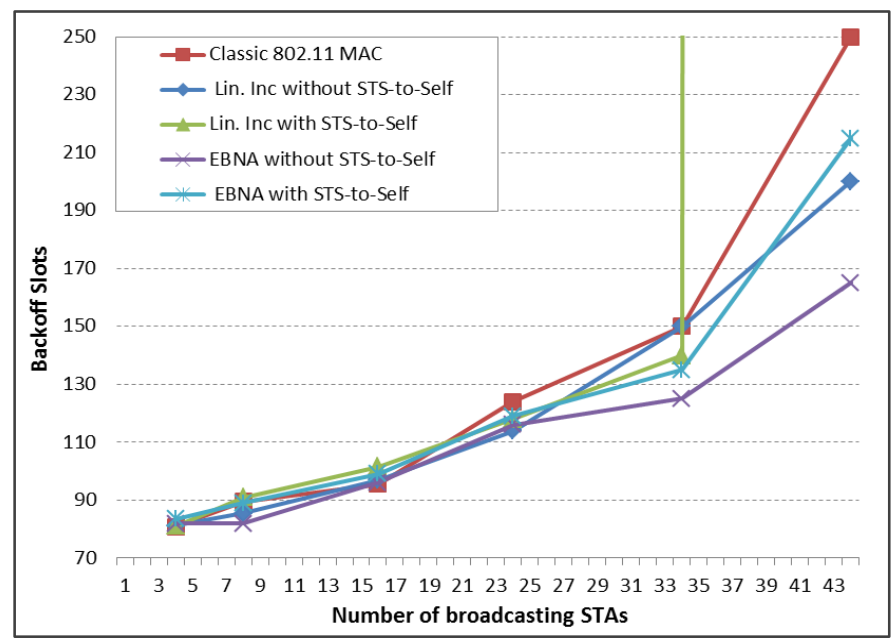

Fig. 13. Number of backoff slots for Unicasting STAs

\section{e) Collisions}

This statistic describes the total number of collisions encountered in the entire network during each simulation. This is not a standard OPNET statistic. In order to obtain this measurement the OPNET wlan_mac process is equipped with a counter which increases every time the collision flag in OPNET is set. The accuracy of this custom statistic was validated using the OPNET collision status statistic which indicate the present of a collision but cannot provide the total number of collisions. It is shown from figure 14 that the lowest 
number of collisions is achieved when the EBNA without CTS-to-Self is used. This is because the EBNA algorithm is designed to minimize collisions in broadcasting STAs.

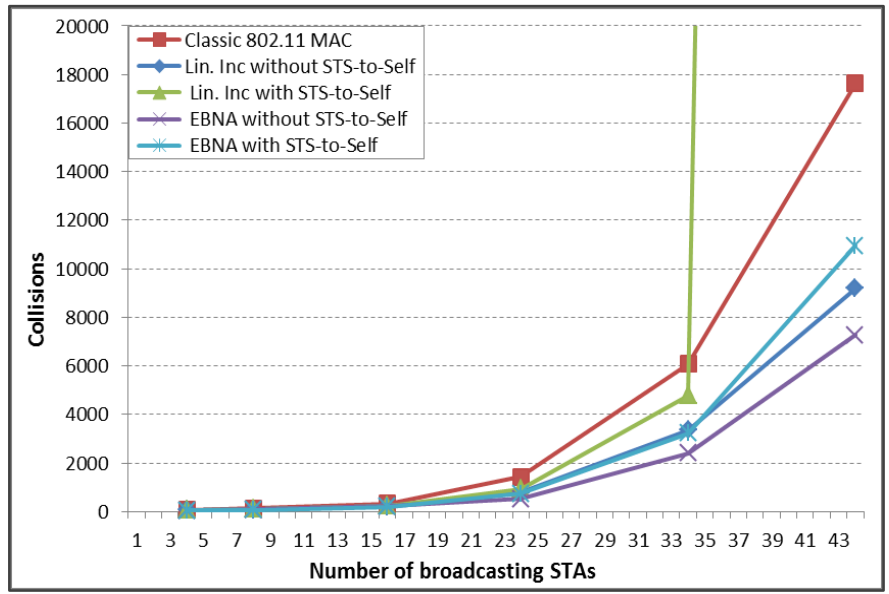

Fig. 14. Number of backoff slots for Unicasting STAs

We can clearly see here that classic 802.11 MAC cause a significant increase of collision when is used as medium access in multiple broadcasting wireless network.

\section{CONCLUSIONS}

In this paper we explore the ability of the modified IEEE 802.11 MAC mechanisms to improve broadcasting performance while are operating in conjunction with a regular wireless network, and also to define the cases were the use of CTS-to-Self-protection mechanism can improve the overall performance of the network. For that reason two alternative MAC mechanisms are proposed. The first one is a linear increase of the $\mathrm{CW}$, directly proportional to the number of broadcasting STAs in the wireless network. The second is simple algorithm which allocates exclusive backoff numbers to each STA that needs to backoff, keeping fairness between STAs during the random backoff process. In addition an extended application of the CTS-to-Self control message is proposed in order to be used for the distribution of network allocation information. All the above techniques have been independently tested, and have demonstrated that they can improve the performance of multiple broadcasting of multimedia type data in wireless ad-hoc networks [14].

In this study all the above modifications are tested in a "real life" scenario in which the modified MAC mechanism coexists in the same BSS with classic IEEE 802.11 devices. The performance of the network is studied as a whole in order to investigate the possibility of modified and classic wireless MAC mechanisms to function together.

The analysis of the results showed that the modified MAC mechanism can coexist with the classic Wi-Fi and also to improve the performance in the case of multiple broadcasting of media data. More specific, a better overall throughput can be achieved while the expected increase of the delay remains in acceptable level. When it comes to the use of CTS-to-Self mechanism, although it slightly improves throughput, it turns that is not an appropriate technique in this "mixed" networks. The significant increase of the traffic that is created by CTS-toSelf messages in combination with the inability of classic 802.11 to manage broadcasting, decrease the performance of the network and in some cases leads to collapse.

Future research can focus in the design of an adoptive EBNA algorithm where the size of the $\mathrm{CW}$ will be dynamically controlled taking into account the current traffic of the wireless network. This will significantly decrease delay and will increase overall performance of broadcasting in the wireless network.

\section{REFERENCES}

[1] Yamaha System Solution "white paper", an introduction to networked audio, Yamaha Comersial Audio 2006, C.Bakker, H.Hamamatsu, T.Harison

[2] AES White Paper: Best Practices in Network Audio, JAES Volume 57 Issue 9 pp. 729-741; September 2009

[3] A. Floros T. Karoudakis, Delivering high-quality audio over WLANs, AES 116th Convention, 2004.

[4] Kevin Gross, Audio Networking, Application and Requirements, JAES Volume 54, No.1/2, January/February, 2006

[5] Nakjung Choi; Yongho Seok; Taekyoung Kwon; Yanghee Choi, "Leader-Based Multicast Service in IEEE 802.11v Networks," Consumer Communications and Networking Conference (CCNC), 2010 7th IEEE, vol., no., pp.1,5, 9-12 Jan. 2010

[6] Fluke networks White Paper, Ensuring 802.11n snd $802.11 \mathrm{a} / \mathrm{b} / \mathrm{g}$ Compatibility, 2011

[7] IEEE Standard, Wireless LAN Medium Access Control (MAC) and Physical Layer (PHY) Specifications, 2007M. Young, the Technical Writer's Handbook. Mill Valley, CA: University Science, 1989.

[8] Brad Williams Tracy Camp, Comparison of Broadcasting Techniques for Mobile Ad Hoc Networks, 3rd ACM international symposium on Mobile ad hoc networking \& computing, 2002

[9] Shiann-Tsong Sheuy, Yihjia Tsai and Jenhui Chen, A Highly Reliable Broadcast Scheme for IEEE 802.11 Multi-hop Ad Hoc Networks, Communications,. ICC 2002. IEEE International Conference, 2002.

[10] Jenhui Chen and Muwen Huang, Broadcast Engagement ACK Mechanism to Support Reliable Broadcast Transmission in IEEE 802.11 Wireless Ad Hoc Networks, Vehicular Technology Conference, 2004.

[11] Xiaomin Ma Xianbo Chen, Performance Analysis of IEEE 802.11 Broadcast Scheme in Ad Hoc Wireless LANs, IEEE Transaction on Vehicular Technology, VOL.57, NO.6, November 2008.

[12] C Chousidis, R Nilavalan, A Floros, Enhancement of IEEE 802.11 in Handling Multiple Broadcasting Audio Data in Wireless ad-hoc Networks, Journal of the Audio Engineering Society, Vol 61, Issue 4, April, 2013

[13] C Chousidis, R Nilavalan, Improving multiple broadcasting of multimedia data traffic in wireless ad-hoc networks, Science and Information Conference 2013.

[14] C Chousidis, R Nilavalan, L Lipa, Expanding the use of CTS-to-Self mechanism to improving broadcasting on IEEE 802.11 networks, IEEE Wireless Communications and Networking Conference (WCNC) 2014, (under review) 